\section{Benign Paroxysmal Positional}

\section{Vertigo (BPPV) and microvasculitis:}

\section{A comparative study between nasal cytology and pathology}

\section{Armone Caruso Arturo ${ }^{1 *}$, Del Prete Salvatore ${ }^{2 *}$, Marasco Daniela², Veronica Viola ${ }^{3}$, Sabato Leo $^{4}$, Andrea Fulgione ${ }^{5}$, Daniele Naviglio ${ }^{3}$ and Monica Gallo ${ }^{6}$}

\author{
'Department of ENT, AIAS Structure of Afragola, Contrada Leutrec snc, 80021 Naples, Italy \\ ${ }^{2}$ Service Biotech s.r.I., Napoli, 80132 Italy \\ ${ }^{3}$ Department of Chemical Sciences, University of Naples Federico II, Naples 80126, Italy \\ ${ }^{4}$ ENT Outpatient Clinic, Scafati Hospital ASL SA
}

${ }^{5}$ Department of Agricultural Science, University of Naples Federico II, Via Università 100, Portici

(NA) 80045, Italy

${ }^{6}$ Department of Molecular Medicine and Medical Biotechnology, University of Naples Federico II, Italy

\section{Abstract}

Purpose: Benign paroxysmal positional vertigo (BPPV) is the most frequent peripheral vertigo syndrome in otoneurological clinical practice and is characterized by short and paroxysmal objective vertigo crises caused by changes in the position of the head on specific planes of space. Secondary microvasculitis is characterized by inflammatory destruction of the small vessels. Starting from this point, this work is based on the research of correlation between microvasculitis (especially secondary), recurrent BPPV and nasal cytology, an aspect, among other things, poorly documented.

Materials and methods: To evaluate the relationships between recurrent BPPV and secondary microvasculitis, nine patients with this disorder, 5 males and 4 females aged between 25 and 40 years were observed (average age 30.6). Non allergic pains in the small joints and in the anamnesis nothing relevant in the gentiles and collaterals: Evaluated with vestibular audiometric examination, nasal cytology and vascular examination with corneal HRT results. Nasal cytology, in all cases, has documented the presence of rare mast cells. The hemodynamics of the microcirculation with a confocal microscope has revealed blood flow alteration in all subjects.

Discussion: Analyzing the results, both the prevalence of the right side and a close relationship between vasculitis and BPPV could be seen.

Conclusion: The study of the nasal mucosa and the research of inflammatory cells could be fundamental for the study of BPPV in which as we have seen the important biochemical role for the onset of these diseases.

\section{More Information}

*Address for Correspondence: Armone Caruso Arturo, Department of ENT, AIAS Structure of Afragola, Contrada Leutrec snc, 80021 Naples, Italy; Tel: +39 3396069421;

Email: armocar@libero.it; saldelp@gmail.com

Submitted: 18 February 2020

Approved: 23 February 2020

Published: 24 February 2020

How to cite this article: Arturo AC, Salvatore D, Daniela M, Viola V, Leo S, et al. Benign Paroxysmal Positional Vertigo (BPPV) and microvasculitis: a comparative study between nasal cytology and pathology. Heighpubs Otolaryngol Rhinol. 2020; 4: 001-005.

\section{DOI: 10.29328/journal.hor.1001017}

Copyright: @ 2020 Arturo AC, et al. This is an open access article distributed under the Creative Commons Attribution License, which permits unrestricted use, distribution, and reproduction in any medium, provided the original work is properly cited.

Keywords: Vasculitis: Mastocites; BPPV; Nasal cytology; Confocal microscope

Check for updates

open access

\section{Introduction}

Benign paroxysmal positional vertigo (VPPB) is the most frequent peripheral vertigo syndrome in otoneurological clinical practice and is characterized by short and paroxysmal objective vertigo crises caused by changes in the position of the head on specific planes of space. The crises have a sudden onset, with rapid intensification up to a plateau maintained from a few seconds to a few minutes, and rapid decrease until disappearance. Vertigo is typically positional, i.e. triggered changes in the position of the head on specific planes of space.

VPPB predominantly affects the female sex in a ratio between 1.6: 1 and 2: 1 [1]; the causes relating to their onset are not fully known, however recent studies have documented a significant statistical correlation between VPPB, especially 
in the recurrent forms, microcirculation and secondary vasculitis [2-4].

Secondary microvasculitis is characterized by inflammatory destruction of the small vessels. This picture involves a modification of vascular homeostasis because, as we will see below, the mast cells, which are present in the endothelium, synthesize, store and secrete both pro and anti-inflammatory molecules, responsible for the state of suffering of the utricular and/or saccular regions with consequent detachment of the otocons.

\section{Molecular composition of the hemolabyrinthine barrier}

The emo-labyrinthine barrier contains a series of enzymes and transporters, which together maintain the necessary extracellular environment of the system.

The mostabundant protein identified in the blood-labyrinth barrier is the subunit ion transporter, $\mathrm{Na}+/ \mathrm{K}+-\mathrm{ATPase} \alpha 1$ and a large number of proteins are metabolic enzymes, including glutathione S-transferase (GST), prosaposin, leukotriene A4 hydrolase -glutamate-oxaloacetate transaminase. LTB4 is a vasoconstrictor that can cause VPPB by changing labyrinthine blood flow. Furthermore, the capillaries of the vascular streak are rich in cell adhesion and tight junction proteins, which is consistent with the labyrinth-blood barrier function [5].

\section{Regulation of the permeability of the hemolabyrinthine barrier (BLB)}

The inner ear has an endothelial barrier of blood tissue in the vascular streak that is as long as the blood-brain barrier. Recently, it has been discovered that the PKC $\eta$ protein regulates the barrier-permeability by interacting directly with $\mathrm{Na}+/ \mathrm{K}+-$ ATPase $\alpha 1$ influencing the state of phosphorylation of occludin.

\section{Materials and methods}

To evaluate the relationships between recurrent BPPV and secondary microvasculitis, nine patients suffering from this disorder were observed at the ENT diagnostic and nasal cytology center of Afragola, 5 males and 4 females aged between 25 and 40 years (average age 30.6). Non allergic pains in the small joints and in anamnesis nothing relevant in the gentiles and in the collaterals, after carrying out privacy and informed consent, we proceeded to: - Vestibular examination, performed with Frenzel glasses. During the examination, spontaneous and positioning nystagmas were sought. - es. Audiometric, performed with Audiometer Resonance R27 a - EG Impedence performed with Impedance meter Resonance 26 m - Nasal cytological examination was performed by collecting nasal mucosa cells on the median part of the inferior turbinate, bilaterally, with nasal-scraping®, which were positioned on an electrostatic charge cytology slide, Superfrost $₫$ Plus Menzel - Gläser ThermoScientific. Staining was carried out according to the Pappenheim method
(3 min in pure May-Grunwald dye; 6 min. In May-Grunwald at 50\%; $1 \mathrm{~min}$. In double-distilled water; $30 \mathrm{~min}$. In Giemsa 1:10 solution). The slide was subsequently covered with a coverslip measuring 24 x $50 \mathrm{~mm} \mathrm{\#} 1$ and observed with the Nikon eclipse 50 i optical microscope, at $100 \mathrm{x}$ magnification in oil immersion. A Nikon DS Fi1 camera with a NIS image acquisition program - Elements D Version 2.30 was used to acquire images under the microscope - Eye examination with study of the fundus and study of the hemodynamic of microcirculation with Heidelberg HRT III confocal microscope corneal module (Figures 1,2).

\section{Results}

All the patients examined showed: at the vestibular examination, everyone had a crisis in progress. Of these, 7 (3 female and 4 male) VPPB of CSP and 2 ( 1 female and 1 male) VPPB of CSL. The prevalence was on the right.

On the audiometric examination, a slight drop on the acute frequencies, between 6000 and $8000 \mathrm{~Hz}$, between 20 and $30 \mathrm{~dB}$. Of these 6 ( 3 females and 3 males) asymmetric and 3 symmetric ( 1 female and 2 males).

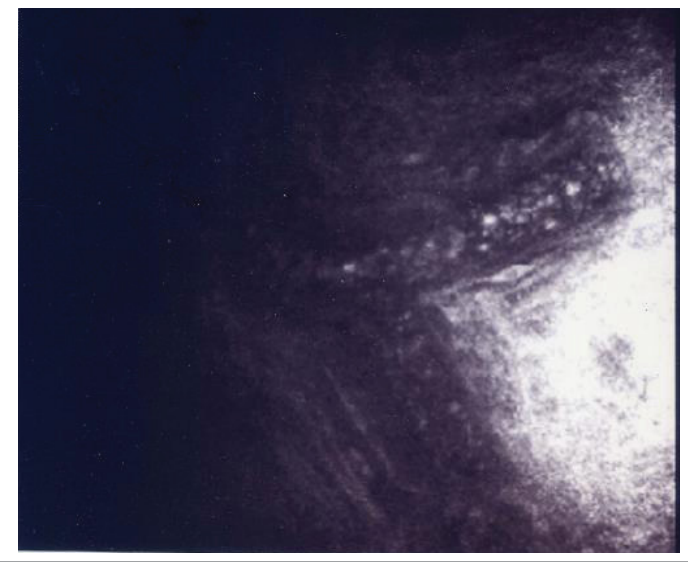

Figure 1: This figures shown the movement of the blood inner in the scleral vessel in the patients with BPPV. The arrows shown the dissection of the scleralmicrovessel.

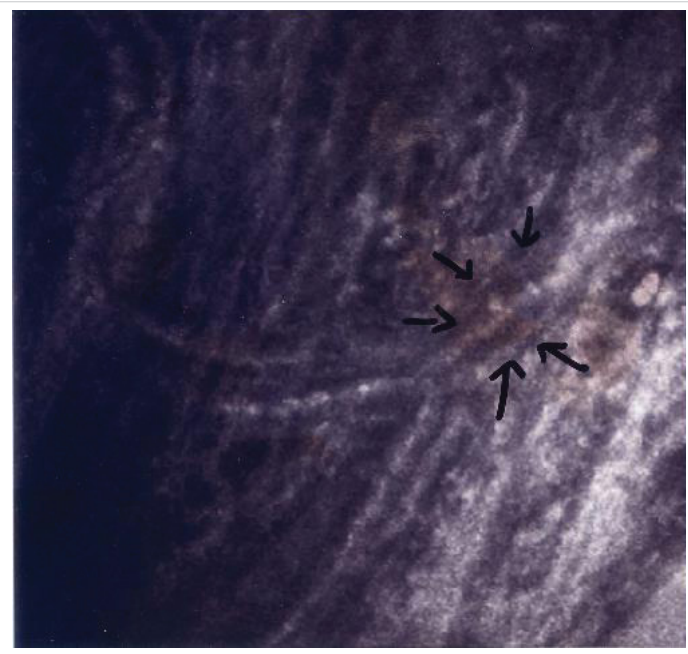

Figure 2: It is possible to see the blood units pass inner the vessel. 
Impedance tests have documented, in all cases, normal tympanograms with the presence of stapedial reflexes within physiological limits.

Nasal cytology, in all cases, has documented the presence of rare mast cells; figure 3 is a cytological nasal scraping, with MayGrunwald coloration, that shows mastocytes or metachromatic granules in nasal mucosa in BPPV patient (Figure 3) The study of the ocular fundus, in all patients documented the presence of suffering of the retinal microcirculation, characterized by micro Drusen only one case of initial anterior uveitis.

The hemodynamics of the microcirculation with a confocal microscope revealed in all subjects alteration of the blood flow, characterized by inversion of the corpuscular arrangement of the elements of the blood and pericytes suffering (Figures $1,2)$.

Immunological investigations were carried out on all subjects to evaluate the presence of secondary sub-clinical vasculitis, with the exception of diabetic pathology which already indicates per se alterations in the functionality of the microcirculation.

All subjects affected by mild mastocytosis had parameters indicative of inflammation of the small vessels characterized by suffering from the pericytes and destructuring of the hemodynamic function (Table 1).

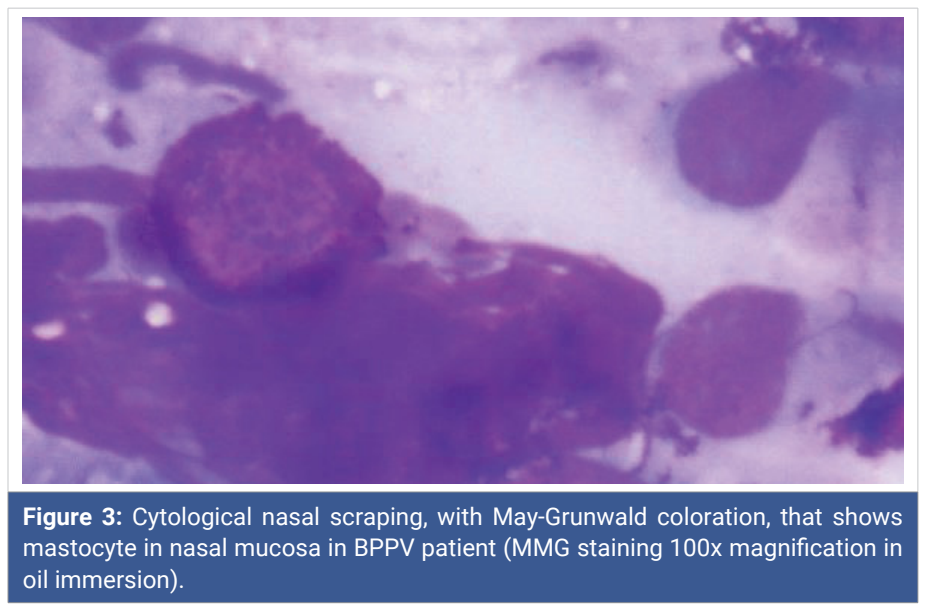

\section{Discussion}

Analyzing the results, both the prevalence of the right side and a close relationship between vasculitis and VPPB could be seen. This aspect is confirmed by international literature [ 6 , 7]. Another relevant condition is given by the slight hearing loss which hypothesizes a suffering of the microcirculation, confirmed by the ophthalmological investigations.

Furthermore, the hearing loss is congruous with the side of the otoconial detachment. This allows us to hypothesize which arterial branch could be affected.

An additional element in this type of study was the observation of the nasal mucosa under a microscope, to assess the presence of any inflammatory cells; and this research was able to confirm the presence of mast cells. These cells can be included in vasculitic and autoimmune diseases [8]. Precisely on inflammatory cells, a close relationship between them and the pathologies [9] has already been documented in a previous communication, which are capable of causing secondary vasculitis. The investigations carried out documented in all the subjects examined were affected by this clinical picture, in the context of which the nasal mast cells can have a role of marker, because these cells play an important role in the onset of immune-mediated diseases [10].

Mast cells (MC) derive from CD [7] positive hematopoietic progenitors in the bone marrow and are released into the circulation, from where they migrate to vascularized tissues or serous cavities. In addition, cytokines such as interleukin IL-3, IL-4, IL-9, IL-10, nerve growth factor (NGF), some chemokines and retinoid acid can regulate the differentiation of MC27. MCs are widely distributed in vascularized tissues. Based on their location and protease content, MCs are divided into two main subtypes:

1) MCs of the mucous membranes (MMCs) associated with the epithelium of the lung and gastrointestinal tract and which express the chymases MMCP- 1 and MMCP- 2 .

2) iMCs of connective tissue (CTMCs) present in the intestinal submucosa, in the peritoneum and in the skin.

Table 1: Cytological nasal scraping, with May-Grunwald coloration that shows mastocyte in nasal mucosa in BPPV patient (MMG staining $100 \mathrm{x}$ magnification in oil immersion).

\begin{tabular}{|c|c|c|c|c|c|c|}
\hline Pz & SEx & Age & Disease & VPPB & Hearing loss & Cells \\
\hline 1 & $\mathrm{~F}$ & 25 & AR & CSP right & $\begin{array}{l}\text { right: } 25 \mathrm{dbHL} \text { a } 8000 \mathrm{~Hz} \\
\text { left: normal hearing }\end{array}$ & 1 mast \\
\hline 2 & $\mathrm{~F}$ & 30 & AR & CSP right & $\begin{array}{l}\text { right:30 dbHL a } 6000-8000 \mathrm{~Hz} \\
\text { left: normal hearing }\end{array}$ & 2 mast \\
\hline 3 & M & 28 & AR (uveities) & CSP right & $\begin{array}{l}\text { right: normal hearing } \\
\text { left: } 25 \mathrm{dbHL} \text { a } 8000 \mathrm{~Hz}\end{array}$ & 3 mast \\
\hline 4 & M & 31 & AR & CSP sin & $25 \mathrm{dBHL}$ a $6000-30 \mathrm{dBHL} 8000 \mathrm{HZ}$ simm & 1 mast \\
\hline 5 & $\mathrm{~F}$ & 40 & Beçhet & CSL right & $25 \mathrm{dbHL}$ a 8000 Hzsimm. & Metachromatic granules \\
\hline 6 & M & 34 & Type 1 diabetes mellitus & CSP right & $25 \mathrm{dBHL}$ a $6000-30 \mathrm{dBHL} 8000 \mathrm{HZ}$ & 4 mast \\
\hline 7 & M & 31 & Type 1 diabetes mellitus & CSL right & $\begin{array}{l}\text { right: } 25 \mathrm{dbHL} \text { a } 8000 \mathrm{~Hz} \\
\text { left: normal hearing }\end{array}$ & 3 mast \\
\hline 8 & M & 28 & Type 1 diabetes mellitus & CSP right & $30 \mathrm{dbHL}$ a $6000-8000 \mathrm{Hzsimm}$ & 1 mast \\
\hline 9 & $\mathrm{~F}$ & 29 & Psoriasic & CSP right & $\begin{array}{l}\text { left: } 30 \mathrm{dbHL} \text { a } 6000-8000 \mathrm{HL} \\
\text { right: normal hearing }\end{array}$ & 4 mast \\
\hline
\end{tabular}


CTMCs are characterized by the expression of the mMCP-4 chimase, an elastinolytic enzyme (mMCP-5) and two tryptases (mMCP-6 and mMCP-7) and carboxypeptidase 3 (CPA3).

In humans, MCs are classified as MCTs, which express only tryptases, or MCTCs, which express tryptases, chimases and CPA3. The main mechanism of MC activation is through the IgEdependent antigen aggregation of the high affinity IgE receptor, FceRI, which triggers the degranulation of the MC. However, MCs can also be activated differently from complement system fractions (anaphylotoxins), immunoglobulin-free light chains, hormones, neuropeptides and Toll Like receptors (TLR). MC activation can induce the release of various biologically active products. These include preformed molecules such as histamine, serotonin, TNF-a, quinines and proteases stored in secretory granules. Leukotrienes (LT), prostaglandins and activated platelet factor (PAF) are synthesized during the activation of $\mathrm{MC}$ by arachidonic acid. In addition, a number of cytokines (IL-1, 2, 5, 6, 8, 9, 13, 17, TNF and TGF- $\beta 1$ ) chemokines (CCL1, CCL2, CCL3, CCL3L1 CCL4, CCL5, CCL7, CCL8, CCL11 and CXCL2) and growth factors (VEGF, PDGF, bFGF, EGF, IGF-1 and NGF) are synthesized de novo and released several hours after their stimulation. MCs are no longer recognized only as provoking allergies, but also have many homeostatic functions, such as blood flow and coagulation, contraction of smooth muscles and peristalsis of the intestine, mucosal secretion, wound healing, as well as regulation of innate and adaptive immune responses, peripheral tolerance and autoimmunity [8].

Mast cell activation in small vessels Mast cells play a complex role in vascular homeostasis since they synthesize, store and secrete both pro and anti-inflammatory molecules. This phenomenon is best demonstrated in human and animal studies on ANCA-associated vasculitis. A mouse model has been developed that mimics the glomerulonephritis observed in human vasculitis associated with ANCA in which mice are immunized with MPO followed by passive transfer of lowdose basal membrane anti-glomerular antibodies, which therefore induces focal segmental glomerulonephritis. Some authors, using this model, have shown that mast cell deficient mice (KitW-sh/W-sh) show more CD4 + anti-MPOCellule T, a stronger delayed hypersensitivity response to MPO and more severe glomerulonephritis than mice wild. In addition, mast cell deficient mice express fewer regulatory $\mathrm{T}$ cells (Tregs) and reduced IL-10 production from lymph nodes. Reconstitution of mast cells from wild-type control, but not from IL-10 deficient mast cells, significantly increased Treg numbers and attenuated the severity of glomerulonephritis.

Subsequent work by the same group of researchers using human kidney biopsies from patients with positive MPO-ANCA and focal segmental glomerulonephritis demonstrated a higher density of interstitial mast cells (degranulating and spindleshaped mast cells) compared with disease controls (thin basement membrane disease) or disease of minimal change in adults). Mast cells were prominent IL-17 producers in the human kidney based on biopsy assessment. Administration of disodium cromoglycate (DSCG), a mast cell stabilizing agent that acts by affecting the calcium channels and inhibiting mast cell degranulation, attenuated $\mathrm{T}$ cell responses with associated decreases in the production of gamma interferon (IFN $\gamma$ ) and IL-17A promoting the IL-10 production. In addition, DSCG inhibited the development of glomerulonephritis and the presence of mast cells within the kidneys. Administration of DSCG in mast cell deficient mice had no effect on IFN $\gamma$, IL-17A or on the development of glomerulonephritis by providing evidence that these effects are guided through DSCG-induced stabilization ofmastcells. Whether mastcells stimulate or inhibit the immune system depends on the physiological environment of the mast cells and the microenvironment in which they reside. Mast cells release preformed mediators immediately after immunity (eg antigen-IgE complexes, complement and immune aggregates) and non-immune (eg radiocontrast, drugs, exercise, and cold air) induced degranulation. Mast cells can also be activated via TLR4 to synthesize and release a variety of pro-inflammatory mediators including TNF $\alpha$, IL-6, IL-1 and IL-13. Interestingly, various stimuli have been reported to prevent mast cell degranulation or to generate anti-inflammatory mediators. For example, stimulation of vitamin D receptors causes mast cells to release IL-10 without causing degranulation. Similarly, DSCG appears to promote IL-10 mast cell production by inhibiting degranulation. In the mouse model associated with ANCA glomerulonephritis, DSCG was able to attenuate the Th1 and Th17 pathways, which play a dominant role in the pathogenesis of ANCA-associated vasculitis. It is not clear whether this is mainly mediated by the inhibition of mast cell degranulation or by the production of IL-10. The direct effects of mast cells on other innate immune cells, such as neutrophils, in ANCA vasculitis are not clearly defined. Also, like neutrophils, mast cells can produce antimicrobial extracellular traps (MCET). The effects of MCET on the pathogenesis of small vessel vasculitis have yet to be explored [8].

Mast cells and vasculitis includes a diverse group of conditions characterized by inflammation and necrosis of blood vessels which leads to occlusion of vessels and ischemia of tissues. Small vessel vasculitis can be caused by IC-mediated inflammation as in the case of anti-glomerular basement membrane disease, IgA vasculitis (Henoche-Schonlein purpura) and vasculitides secondary to systemic immune system diseases such as SLE, dysproteinemias, cryoglobulinemias and chronic infections. Vasculitis can also be induced by antibodies to auto antigens such as small vessel vasculitis associated with the anti-neutrophil cytoplasmic antibody (ANCA), which includes granulomatosis with polyangiitis (originally Wegener's granulomatosis), microscopic polyangiitis and eosinophilic granulomatosis with polyangiitis syndrome) The anti-neutrophil cytoplasmic autoantibodies found in ANCA vasculitis are mainly directed against the proteins of the azurophyl neutrophil granules proteinase 3 (PR3) and myeloperoxidase (MPO). 
The inoculation of mice with hybridomas that produce monoclonal IgG rheumatoid factors induces the development of a vascular cell-leukocytoclastic caused by cryoglobulin deposition. The binding of IC by FcR positive cells stimulates the secretion of inflammatory mediators that recruit polymorphonuclear cells (PMN), which damage blood vessels. In this model of hypersensitivity angiitis, vascular inflammation depended on the MC recognition of IC by Fc $\gamma$ RIII and the release of preformed TNF- $\alpha$ stored in its granules.

The administration of mercuric chloride $(\mathrm{HgCl} 2)$ in Brown Norway rats causes Th2-induced autoimmunity characterized by high concentrations of IgE and production of multiple IgG autoantibodies in MPO. Animals develop polyarthritis and a leukocytoclastic vasculitis that primarily affects the intestines. Vasculitis in this model has some similarities with human Churg-Strauss syndrome. It has been suggested that MC has a role in the pathogenesis of early disease, which is independent of $\alpha \beta \mathrm{T}$ cells and neutrophils - Non-toxic concentrations of $\mathrm{HgCl} 2$ may induce the release of CTMC mediators and cause up regulation of mRNA expression IL-4 in vitro. Furthermore, the administration of $\mathrm{HgCl} 2$ induced an increase in the $\mathrm{MC}$ protease in the serum rat II (RMCP II) and a reduction of the positively colored toluidine blue cells (interpreted as MC degranulation), indicating MC activation in vivo. In addition, the use of MC G63 and doxantrazole stabilizing agents resulted in an improvement of early cecal vasculitis caused by $\mathrm{HgCl} 2$. On the other hand, MC represented a protective role in the development of necrotizing focal glomerulonephritis (GN) in ANCA-associated vasculitis. The deficient $\mathrm{W}$-sh Kit MC mice developed enhanced anti-MPO GN, characterized by greater kidney injury compared to intact C57BL / 6 MC mice. Furthermore, the reconstitution of mice with attenuated glomerular damage derived from $\mathrm{MC}$ in the bone marrow is indicated by fewer abnormal glomeruli and a lower fibrin deposition. It has been suggested that MC migrated from MPO presentation sites to draining LN where they could modulate the function of regulatory T cells through IL-10 secretion, and these cells, in turn, would reduce the ability of MPO T effector cells to produce proinflammatory cytokines [8].

\section{Conclusion}

VPPB is undoubtedly a pathology considered benign, but if relapsed can be a sign of a much more important pathology. The study reported in this review, even if conducted on a limited number of patients, has not only allowed to better investigate this clinical picture, but also to make an early diagnosis of secondary microvasculitic forms. In particular, the study of the nasal mucosa could be a sort of litmus test for the research of inflammatory cells, which as we have seen, have an important biochemical role for the onset of these diseases. In addition, the study of conjunctival capillaries in vivo could allow not only to objectively diagnose the pathology of the microcirculation, but also, together with the observation of mast cells of the mucous membranes, to monitoring the pathology.

\section{Acknowledgment}

The authors are grateful to Service Biotech laboratories, the ENT Department of AIAS of Afragola, Department of Molecular Medicine and Medical Biotechnology, University of Naples Federico II Department of Chemical Sciences, University of Naples Federico II, Department of Agricultural Science, University of Naples Federico II.

\section{References}

1. Baloh RW, Honrubia V, Jacobson K. Benign positional vertigo: clinical and oculographic features in 240 cases. Neurology. 1987; 37: 371-378. PubMed: https://www.ncbi.nlm.nih.gov/pubmed/3822129

2. Messina A, Casani AP, Manfrin M, Guidetti G. Italian survey on benign paroxysmal positional vertigo. Acta Otorhinolaryngol Ital. 2017; 328: 335. PubMed: https://www.ncbi.nlm.nih.gov/pubmed/28872163

3. Goto F, Hayashi K, Kunihiro T, Ogawa K. The possible contribution of angiitis to the onset of benign paroxysmal positional vertigo (BPPV). Int Tinnitus J. 2010; 16: 25-28.

PubMed: https://www.ncbi.nlm.nih.gov/pubmed/21609909

4. Amor-Dorado JC1, Llorca J, Costa-Ribas C, Garcia-Porrua C, GonzalezGay MA. Giant cell arteritis: a new association with benign paroxysmal positional vertigo. Laryngoscope. 2004; 114: 1420-1425.

PubMed: https://www.ncbi.nlm.nih.gov/pubmed/15280720

5. Shi X. Physiopathology of the cochlear microcirculation. Hear Res. 2011; 2: 10-24

PubMed: https://www.ncbi.nlm.nih.gov/pubmed/21875658

6. Guidetti G, Manzoni D, Galletti G. Il contributo della manovra di Semont alla diagnosi ed alla risoluzione delle vertigini parossistiche da posizionamento. Rivista critica di 310 casi. Acta Otorhinolaryngol Ital. 1988; 8: 488-493.

7. Amor-Dorado JC, Gonzalez-Gay MA. Audiovestibular Manifestations in Systemic Vasculitis: an Update In Vasculitis/Book.

8. Wesing LA, Câmara NOS, Pereira FV. Relationship between Mast Cells and Autoimmune Diseases. Austin J Clin Immunol. 2014; 1: 1016.

9. Caruso AA, Grassia R, Capasso, P, Orabona CA. Is the study of nasal cytology a prognostic index of autoimmune disease? Preliminary notes. Attidel 100th National Congress SIO 2013.

PubMed: https://www.ncbi.nlm.nih.gov/pubmed/8216642

10. Johnston B, Burns AR, Kubes P. A role for mast cells in the development of adjuvant-induced vasculitis and arthritis. Am J Pathol. 1998; 152: 555-563.

PubMed: https://www.ncbi.nlm.nih.gov/pubmed/9466582 